submitted to PRL

\title{
Elastic Energy, Fluctuations and Temperature for Granular Materials
}

\author{
Lou Kondic ${ }^{(1)}$ and R. P. Behringer ${ }^{(2)}$ \\ (1) Department of Mathematical Sciences $\&$ Center for Applied Mathematics 85 Statistics \\ New Jersey Institute of Technology, Newark, NJ 07102 \\ and \\ ${ }^{(2)}$ Department of Physics $\&$ Center for Nonlinear and Complex Systems \\ Duke University, Durham NC, 27708-0305
}

(Dated: July 1, 2003)

\begin{abstract}
We probe, using a model system, elastic and kinetic energies for sheared granular materials. For large enough $P / E_{y}$ (pressure/Young's modulus) and $P / \rho v^{2}(P /$ kinetic energy density) elastic dominates kinetic energy, and energy fluctuations become primarily elastic in nature. This regime has likely been reached in recent experiments. We consider a generalization of the granular temperature, $T_{g}$, with both kinetic and elastic terms and that changes smoothly from one regime to the other. This $T_{g}$ is roughly consistent with a temperature adapted from equilibrium statistical mechanics.
\end{abstract}

PACS numbers: 45.70.-n,05.40.-a,64.70.pf

We explore the role of elasticity in the energy and energy fluctuations of sheared dense granular systems. For dilated gas-like granular states, energy fluctuations are frequently described in terms of a temperature, defined as the fluctuating part of the kinetic energy, $T_{k} \equiv m<$ $v^{2}>/ 2$. Here, $v$ is the local random component of the velocity. This definition is predicated on assumptions such as molecular chaos, absence of correlations, and short-lived collisions, that do not always apply. For dense systems, a very different concept, Edwards entropy, has been proposed [1]. This quantity is the logarithm of the number of jammed configurations consistent with all constraints on the system, and the Edwards temperature is $T_{E}^{-1}=\partial S_{E} / \partial V$, where $V$ is the system volume.

Both of these pictures assume that minimal energy is stored in compressional modes of the particles. This assumption is valid when the pressure is small compared to the Young's modulus, $E_{y}$. However, there are situations when this need not be the case. The main goal of this letter is to analyze via discrete element simulations (DES) the storage of energy and energy fluctuations for dense granular material subject to shearing.

In order to better establish a context, we estimate the relative importance of kinetic and elastic energy for a simplified system. We imagine that spherical particles with a typical velocity, $v$, are subject to an applied force, $L$, at each contact. For simplicity, assume at first just a pair of opposing contacts, so that there is an effective pressure, $P=L / A$, where $A=\pi R^{2}$, and $R$ is the radius of a sphere. The elastic energy per contact (assuming a Hertz-Mindlin contact law) is $\epsilon \propto L^{5 / 3}$. The ratio of elastic to kinetic energy for a particle is $R_{E}=\epsilon / K=$ $C\left(P / E_{y}\right)^{2 / 3}\left(P / \rho v^{2}\right)$, where $C$ is an $O(1)$ constant that depends on the Poisson ratio, $E_{y}$ is the Young's modulus, and $\rho$ is the bulk density. By multiplying by the number of contacts per particle, it is possible to generalize this to a more realistic situation. When $R_{E}$ is small, elastic energy is irrelevant, and vice versa when it is large, it should be included in a description of the system.

We now consider $R_{E}$ for two representative cases. For the experiments of 2], $E_{y}=5 \mathrm{MPa}$, typical $P \sim 160 \mathrm{~Pa}$, and $\rho=1.2 \mathrm{~g} / \mathrm{cm}^{3}$. Typical speeds ranged over $6 \times$ $10^{-4} \mathrm{~m} / \mathrm{s} \leq v \leq 2 \times 10^{-2} \mathrm{~m} / \mathrm{s}$, so that $0.3 \leq R_{E} \leq 4 \times 10^{2}$. For glass spheres [3],$E_{y}$ is larger by roughly a factor of 5000. Assuming densities $\rho \sim 2 \mathrm{~g} / \mathrm{cm}^{3}$, and pressures corresponding to the base of a column $10 \mathrm{~cm}$ high in a gravitational field, $R_{E} \simeq 1$ for velocities of a few $\mathrm{mm} / \mathrm{s}$.

Hence for slowly sheared dense granular systems, there exist velocity regimes for which elastic energy is the dominant mode of energy storage. In such a setting, neither $T_{k}$ nor $T_{E}$ is likely to provide a good measure of the random nature of the system.

In this context, we propose an extension of granular "temperature" that contains information on fluctuations of the elastic energy, and then compare this extension to a relation for temperature drawn from statistical mechanics. (A somewhat similar approach of applying equilibrium statistical theory has been taken in recent works on foams [4] and granular systems [5].) To carry out this exploration, we use DES of 2D particles that are subject to plane shear and (possibly) compression.

The generalization of "temperature" that we consider is based on the classical idea that for a lattice of elastic particles, the average fluctuating energy/particle is $3 k_{B} T$. Using this as a heuristic guide, we define a generalized temperature that is roughly $T_{g}=m\left\langle v^{2}\right\rangle$ $/ 2+k<x^{2}>/ 2$, where $v$ corresponds to the fluctuating part of the velocity, and $x$ to the fluctuating part of the compression of a particle. Note that this definition provides a simple bridge between the extremes of a gas-like state and a highly compressed slowly evolving state.

In the simulation, particles are confined between two inpenetrable straight parallel boundaries, as sketched in Fig. 13. The top boundary, which is 50 mean particle diameters $\left(d_{m}\right)$ long, moves at a steady speed, and induces shearing in the system. The boundary conditions in the shearing direction are periodic. This system avoids the nonuniformity that characterizes physical experiment, which typically exhibit shear bands [2]. Although we could have chosen to use the even simpler 
Lees-Edwards conditions, we have concentrated on the present model so as to explore the influence of boundaries, typically present in physical experiments.

These simulations closely follow the soft-disk/sphere model to describe various granular systems (see [7] and references therein). Here, we concentrate on twodimensional polydisperse disks in a zero- $g$ environment. The walls are made of identical particles that are rigidly attached. Forces between the particles have a normal component given by $\mathbf{F}_{N}=\left[k_{f} x-\gamma_{N} \bar{m}\left(\mathbf{v}_{i, j} \cdot \hat{\mathbf{n}}\right)\right]$ where $k_{f}$ is a force constant, $r_{i, j}=\left|\mathbf{r}_{i, j}\right|, \mathbf{r}_{i, j}=\mathbf{r}_{i}-\mathbf{r}_{j}$, $\hat{\mathbf{n}}=\mathbf{r}_{i, j} / r_{i, j}, d=\left(d_{i}+d_{j}\right) / 2, d_{i, j}$ are the diameters of the particles $i$ and $j, x=d-r_{i, j}$ is the compression, $\mathbf{v}_{i, j}=\mathbf{v}_{i}-\mathbf{v}_{j}, \bar{m}$ is the reduced mass, and $\gamma_{n}$ is the damping constant related to the coefficient of restitution, $e_{n}$. The parameters represent photoelastic disks [2]; in particular, $\gamma_{n}$ corresponds to $e_{n}=0.5$. The tangential force is given by $\mathbf{F}_{S}=\operatorname{sign}\left(-v_{r e l}^{t}\right) \min \left(\gamma_{s} \bar{m}\left|v_{r e l}^{t}\right|, \nu_{k}\left|\mathbf{F}_{N}^{c}\right|\right) \hat{\mathbf{s}}$ where $v_{r e l}^{t}$ is the relative velocity in the tangential direction $\hat{\mathbf{s}}, \gamma_{s}=\gamma_{n} / 2$ and $\mu_{k}$ is the coefficient of friction between the particles. The equations of motion are then integrated using a 4th order predictor-corrector method. Additional simulations (to be presented elsewhere) show that variation of the parameters or the force model modify only details of the results.

The simulations are performed with approximately 2000 polydisperse particles, with a radius variability of $10 \%$. While polydispersity is important to avoid crystallization, the details of the size distribution are not: the results are very similar for different ranges of particle sizes, or a bidisperse distribution. Particles are initially placed on a lattice, given random velocities, and the system is then very slowly compressed to a desired volume fraction, $\nu$. The results that follow use $t$, the time it takes the shearing wall to travel once across the domain, as a time scale, and $l / t\left(l=50 d_{m}\right)$ as a velocity scale.

The quantities below are calculated using space-time averaging. Thus, the system is divided into cells, and averaged quantities are calculated for each cell. In particular, the kinetic temperature is defined by

$$
T_{k}=\frac{1}{2}\left[\left\langle m\left(u^{\prime}\right)^{2}\right\rangle+\left\langle m\left(v^{\prime}\right)^{2}\right\rangle+\frac{\beta}{4}\left\langle m\left(d_{p} \omega^{\prime}\right)^{2}\right\rangle\right],
$$

where $u, v$ are the components of particle velocity, $d_{p}$ is the diameter of a particle, $m$ is its mass $\left(m \sim d_{p}^{2}\right)$, $\beta=1 / 4$ for disks, and $\omega$ is the angular velocity. The primed averages are defined with zero mean, e.g, $\left\langle u^{\prime} v^{\prime}\right\rangle=$ $\langle u v\rangle-\langle u\rangle\langle v\rangle$.

The elastic energy is obtained by averaging per collision, not per particle. The difference between the two is significant for dense granular systems considered here, since particles typically experience multiple collisions. If $x_{j, c}$ is the compression of particle $j$ due to the collision $c$, then our definition of the elastic energy in cell $l$ is

$$
E_{e, l}=\frac{1}{N_{t} n_{l}} \frac{k_{f}}{2} \sum_{k=1}^{N_{t}} \sum_{j=1}^{n_{l}} \sum_{c=1}^{n_{c, j}}\left[x_{j, c}\right]^{2},
$$

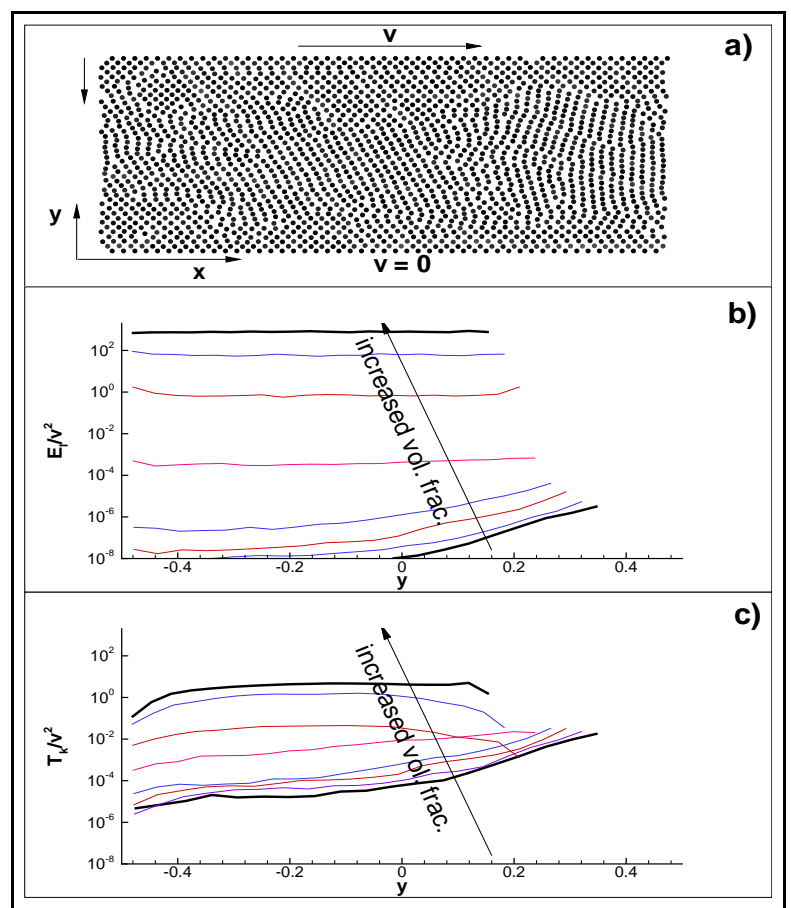

FIG. 1: (a) The geometry of the simulations (only centers of the particles are shown). (b,c,) Elastic energy and kinetic temperature, scaled by $v^{2}$, as functions of the distance from the shearing wall, $y$. The shearing wall is to the right, and the shearing is in the out-of-plane direction $(v=0.1)$.

where $n_{l}$ is the number of particles in cell $l$ at a given time, and $\bar{n}_{l}$ is the average number of particles during the period of $N_{t} \gg 1$ time steps (in practice, the averaging time scale is sufficiently short so that to a high degree of accuracy $n_{l}=\bar{n}_{l}$ ). Definition (1) ignores the energy used to overcome friction, and its form clearly depends on the form of the normal force, e.g., for a $3 \mathrm{D}$ systems, for which a Hertzian interaction law $\left(F_{N} \sim x^{3 / 2}\right)$ is more appropriate, $E_{e, l}$ is of different form as well.

Figure 1 shows the elastic energy and kinetic temperature (scaled by the square of the shearing velocity $v$, and by the average mass of a particle) vs. distance, $y$, from the shearing wall. For these simulations, $\nu$ is continuously increased by (slow) compression. $\nu$ increases from $65 \%$ (bottom) to $90 \%$ (top) (note that in 2D, random close packing and cubic close packing correspond to about $85 \%$ and $2 \pi / \sqrt{3} \approx 90 \%$, respectively). Clearly, as $\nu$ is increased, there is a transition region (about $\nu_{c}=80 \%$ ) where the energy stored in the internal degrees of freedom (elastic energy) becomes more relevant than the kinetic energy (see also Fig. (2). The $y$-dependence of the results is rather weak and becomes even weaker for higher $\nu$ 's. Hence, hereafter, we ignore the $y$-dependence and use system averages of locally computed quantities.

As $\nu$ increases, the energy is mainly elastic, and $T_{k}$ loses its relevance. In order to have a quantity that might play the same role as $T_{k}$ in a dense granular system we 
propose a generalized granular 'temperature' by

$$
T_{g}=T_{k}+T_{e},
$$

as a sum of $T_{k}$, and the 'elastic' part $T_{e} . T_{e}$ is defined as the mean fluctuation of elastic energy, in a manner similar to $T_{k}$, which is the mean fluctuation of kinetic energy. This definition follows the classical statistical mechanics result where the mean fluctuations in the combined elastic and kinetic energy of an oscillator are proportional to the temperature. However, unlike this classical case, there is no reason to expect equipartition between elastic and kinetic modes. Rather, the ratio $T_{e} /\left(T_{e}+T_{k}\right)$ varies from 0 in the dilute limit, to 1 in the dense limit.

The definition of $T_{e}$ requires some care due to multiple collisions. One simple and natural definition that is consistent with the classical statistical definition of temperature for an oscillator is as follows. We first define the average elastic energy per particle in cell $l$ as

$$
\left\langle E_{e, l}\right\rangle=\frac{k_{f}}{2} n_{c}\left\langle x_{l}\right\rangle^{2}=\frac{k_{f}}{2} n_{c}\left[\frac{1}{N_{t} \bar{n}_{l} n_{c}} \sum_{k=1}^{N_{t}} \sum_{j=1}^{n_{l}} \sum_{c=1}^{n_{c, j}} x_{j, c}\right]^{2},
$$

where $\left\langle x_{l}\right\rangle$ is the average compression per collision, and $n_{c}$ is the average number of collisions per particle. Then,

$T_{e, l}=\frac{k_{f}}{2} n_{c}\left\langle\delta x^{2}\right\rangle=\frac{k_{f}}{2} n_{c}\left\langle\left(x_{j, c}-\left\langle x_{l}\right\rangle\right)^{2}\right\rangle=E_{e, l}-\left\langle E_{e, l}\right\rangle$,

where the last equality easily follows using (11) and (3).

Figure 2 shows $T_{k}, T_{e}$, and $\left\langle E_{e}\right\rangle$ vs. time, for four different $\nu$ 's. Unlike the results of Fig. [1 these results are obtained after shearing for long times at fixed $\nu$ 's. For higher $\nu$ 's, clearly $T_{e} \gg T_{k}$. Interestingly, there are rather large fluctuations of the results with $\nu$ just above $\nu_{c} \approx 80 \%$, corresponding to the regime where elastic energy becomes predominant. Also, experiments have indicated a phase transition for comparable densities which may be related [2] (see also [8]). Generally, one might expect both glassy and/or jamming phenomena to dominate this regime, a point that we will explore elsewhere.

We further interpret $T_{g}$ by comparing it to an alternative definition from statistical mechanics. We consider, among various possibilities, the standard relation [6]

$$
\frac{d U}{d T}=\frac{\delta U^{2}}{T^{2}},
$$

where $U$ is the total energy in the (usually conservative) system, $T$ is the temperature, and $\delta U^{2}=\left\langle U^{2}\right\rangle-\langle U\rangle^{2}$. We use $U=E_{k i n}+\left\langle E_{e}\right\rangle$, and ask whether it makes sense to define $T=T_{g}=T_{k}+T_{e}$. To check this idea, we now think of (5D) as a defining equation for $T_{m}=\sqrt{\delta U^{2}} /\left(d U / d T_{g}\right)$ ( here $T_{m}$ stands for 'model temperature'). The agreement between $T_{m}$ and $T_{g}$ will provide some indication of the utility of the definition for $T_{g}$. Note that we should not expect perfect or possibly even any agreement, since we are considering a strongly

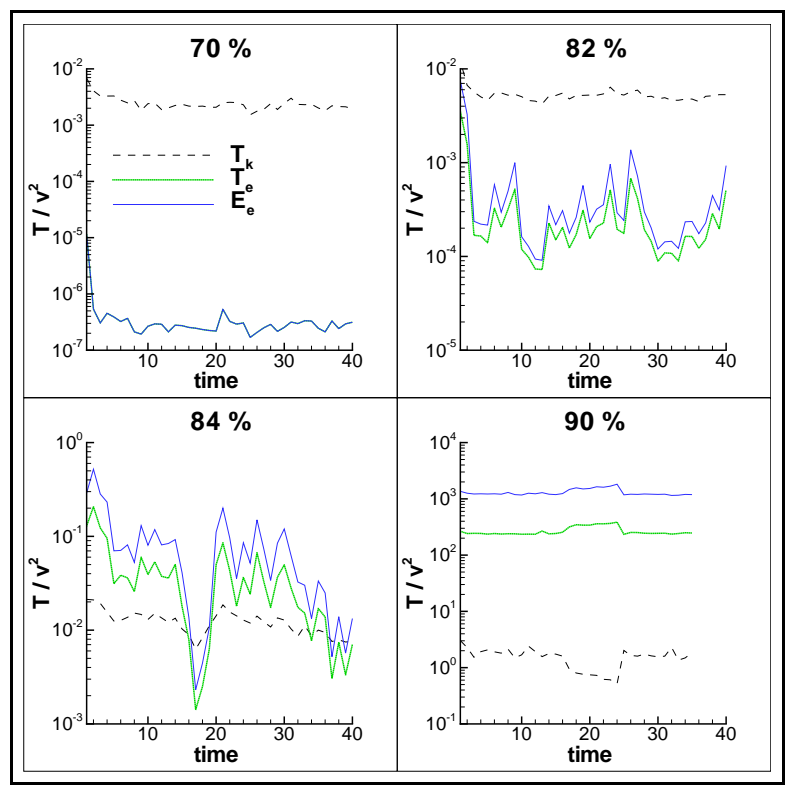

FIG. 2: Elastic energy, kinetic and elastic temperatures for various $\nu$ 's, scaled by $v^{2}$.

dissipative system far from equilibrium. We emphasize that there are no fitting parameters and that the results that follow are obtained directly from simulations.

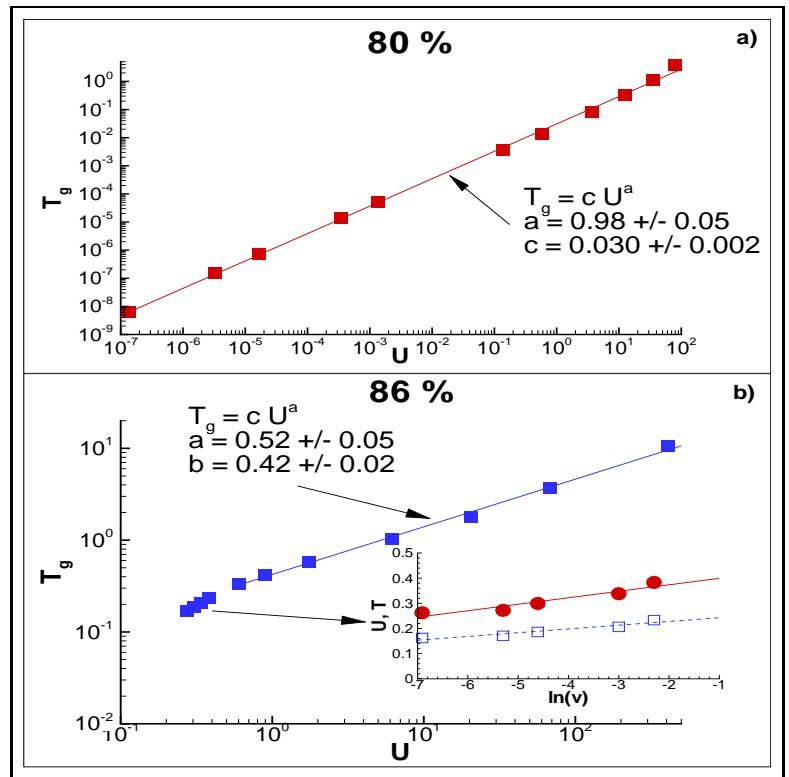

FIG. 3: Generalized temperature versus average energy for two different $\nu$ 's (increased $U$ corresponds to increased shearing velocity $v$ ). The lines are the least square fits to the data. In the inset of b), $U$ (solid line, filled circles) and $T_{g}$ (broken line, squares) are plotted versus $\ln (v)$ for slow shearing. 


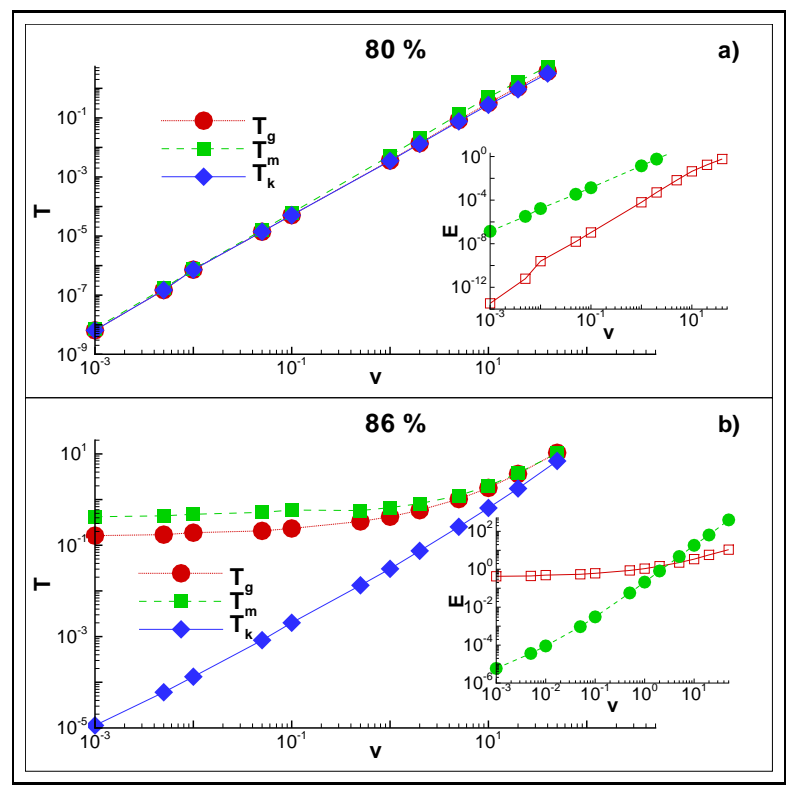

FIG. 4: Generalized, kinetic, and 'model' temperature (see the text and Eq. (5)) for two volume fractions. Error bars (resulting from statistical uncertainty of the results, as well as from the uncertainty introduced by the least square fits from Fig. 31) are approximately represented by the size of the symbols. The insets show kinetic energy (filled circles) and average elastic energy (squares).

Figure [3 shows typical data used to determine $c_{v}=$ $d U / d T_{g}$ needed for $T_{m}$. The range of $U$ 's shown in Fig. 3 corresponds to $0.0001<v<40$. For $\nu=80 \%, U \sim T_{g}$ over almost 10 decades. For $\nu=86 \%, U \sim T_{g}^{a}$, where $a \simeq 2.0$. However, in this case, there is a deviation from the power law fit for slow shearing, and we are limited to a smaller range of $U$ 's, since most of the energy is stored in the system as elastic energy. In order to obtain better data for $U\left(T_{g}\right)$ for slow shearing at higher densities, we determine $U$ and $T_{g}$ as functions of $v$, see Fig. B 3 . We then find to a good approximation that both $U$ and $T_{g}$, are proportional to $\ln (v)$. This logarithmic dependence for slow shearing is, to the best of our knowledge, the first computational confirmation of recent experimental results [9], and will be presented in more detail elsewhere. For our purposes here, it is sufficient to extract $U\left(T_{g}\right)$.

The dependence of $U\left(T_{g}\right)$ is interesting. We note that $U \sim T_{g}$ for $\nu=80 \%$ is similar to a recent simulation of sheared foams [4]. However, the result $U \sim T_{g}^{2}$ for $\nu=86 \%$ and for not too slow shearing $(U>1$, see
Fig. 3b) is striking and deserves some comment. Although the increased role of elastic energy is important here, this does not appear to be the whole story, as seen in the context of Fig. 4 Here, we show that even for this high $\nu$, for fast enough shearing the kinetic energy is still dominant. Our preliminary interpretation is that increased volume fraction contributes significantly to decreased mobility (jamming) of the granular system, therefore reducing the increase of $T_{g}$ with $U$.

Figure 4 contains a summary of the various types of temperatures considered in this study for two $\nu$ 's. As already noted, for $\nu=80 \%$, most of the energy is still kinetic, while for $\nu=86 \%$, elastic energy is essential. For $\nu=80 \%, T_{k}$ is dominant, and also it satisfies the model (5) since $T_{k} \approx T_{m}$. However, for $\nu=86 \%$ and for slow shearing, $T_{k}$ is smaller by 4 orders of magnitude than $T_{g}$ and $T_{m}$. Thus, $T_{k}$ cannot be used to even approximately describe a dense slowly sheared granular system. This difference decreases for higher shearing rates, but only at very high shearing is there good agreement.

The agreement between $T_{g}$ and $T_{m}$ is not perfect, although prefect agreement is not to be expected. These studies demonstrate the clear need to incorporate elastic energy and elastic fluctuations, and that $T_{g}$ has utility as a generalized granular temperature. Nevertheless, there remain many open questions regarding the extent to which the various temperatures serve similar functions to their molecular counterpart. We will present detailed results addressing this type of question elsewhere. Here we note that at least qualitatively one can show that these temperatures can be used in the context of thermal conduction, i.e. the there is a flow of heat from hot to cold regions. We also note that the distributions of both kinetic and elastic energy are strongly non-Gaussian for a significant range of the parameters analyzed here.

There are other possible tests of the proposed generalization, as recently discussed on the context of sheared foams [4]. The ultimate test will be to perform physical experiments where the validity of the proposed concepts can be verified. In the theoretical direction, it will be of interest to relate the generalized granular temperature proposed here to the one resulting from Edwards-entropy based approach.

We acknowledge support by NASA NAG3-2367 and NAG3-2372. RPB acknowledges support by NSF grants DMR-0137119, DMS-0204677 and DMS-0244492.
[1] S. F. Edwards. In A. Mehta, editor, Granular Matter: An Interdisciplinary Approach, 121, Springer,New York 1994.

[2] D. Howell, C. Veje, and R. P. Behringer. Phys. Rev. Lett., 82:5241, 1999.

[3] W. Losert, J.-C. Geminard, S. Nasuno, and J. P. Gollub. Phys. Rev. E, 61:4060, 2000.

[4] I. K. Ono etal. Phys. Rev. Lett., 89:095703, 2002.
[5] H. A. Makse and J. Kurchan. Nature, 415:614, 2002.

[6] D. A. McQuarrie. Statistical Mechanics. University Science Books, Sausalito, 2000.

[7] L. Kondic. Phys. Rev. E, 60:751, 1999.

[8] C. S. Campbell. J. Fluid. Mech., 465:261, 2002.

[9] R. P. Behringer and R. R. Hartley. Nature, 421:928, 2003. 